\title{
Understanding bottom-up continuous hydrothermal synthesis of nanoparticles using empirical measurement and computational simulation
}

\author{
José Sierra-Pallares ${ }^{1}$, Thomas Huddle ${ }^{2}$, Juan García-Serna ${ }^{3}$, Esther Alonso ${ }^{3}$, Fidel Mato ${ }^{3}$, Igor Shvets ${ }^{4}$, \\ Olaf Luebben ${ }^{4}$, María José Cocero ${ }^{3}$, and Edward Lester ${ }^{2}(\varangle)$ \\ ${ }^{1}$ Fluids Engineering Group Energy and Fluid Mechanics Engineering Department, University of Valladolid, Paseo del Cauce 59,47010 \\ Valladolid, Spain \\ ${ }^{2}$ Advanced Materials Research Group, the University of Nottingham, University Park, Nottingham NG7 2RD, UK \\ ${ }^{3}$ High Pressure Process Group. C/Prado de la Magdalena s/n, Department of Chemical Engineering and Environmental Technology, \\ University of Valladolid, 47011 Valladolid, Spain \\ ${ }^{4}$ School of Physics, Trinity College Dublin, College green, Dublin 2, Ireland
}

\author{
Received: 7 June 2016 \\ Revised: 11 July 2016 \\ Accepted: 13 July 2016 \\ (C) Tsinghua University Press \\ and Springer-Verlag Berlin \\ Heidelberg 2016

\section{KEYWORDS} \\ nanoparticle, \\ computational fluid \\ dynamics, \\ synthesis, \\ supercritical, \\ inorganics
}

\begin{abstract}
Continuous hydrothermal synthesis was highlighted in a recent review as an enabling technology for the production of nanoparticles. In recent years, it has been shown to be a suitable reaction medium for the synthesis of a wide range of nanomaterials. Many single and complex nanomaterials such as metals, metal oxides, doped oxides, carbonates, sulfides, hydroxides, phosphates, and metal organic frameworks can be formed using continuous hydrothermal synthesis techniques. This work presents a methodology to characterize continuous hydrothermal flow systems both experimentally and numerically, and to determine the scalability of a counter current supercritical water reactor for the large scale production $\left(>1,000 \mathrm{~T} \cdot\right.$ year $\left.^{-1}\right)$ of nanomaterials. Experiments were performed using a purpose-built continuous flow rig, featuring an injection loop on a metal salt feed line, which allowed the injection of a chromophoric tracer. At the system outlet, the tracer was detected using UV/Vis absorption, which could be used to measure the residence time distribution within the reactor volume. Computational fluid dynamics (CFD) calculations were also conducted using a modeled geometry to represent the experimental apparatus. The performance of the CFD model was tested against experimental data, verifying that the CFD model accurately predicted the nucleation and growth of the nanomaterials inside the reactor.
\end{abstract}




\section{Introduction}

Any application of nanotechnology will generally rely on the effective use of nanomaterials and whether the nanomaterials themselves are fit for the intended purpose [1]. Examples of this include printed electronics, where the ink requires controlled size distributions in high weight loadings without shear thickening properties [2]; lighting and display applications, where quantum dots are used with size control to within two decimal places of a single nanometer [3]; and lab-on-a-chip devices, which use a specific particle size to create plasmon effects [4]. There are many ways to synthesize nanomaterials, and the choice of technique will, in turn, dictate the quality, range, and value of the products, from lowvalue and high-volume materials (e.g., $\mathrm{TiO}_{2}$ in paints from the acid treatment of ilmenite) to high-value and low-volume materials (e.g., graphene production using chemical vapor deposition) $[5,6]$.

A comprehensive review by Santamaria et al. [7] recently highlighted many different synthesis routes for the production of nanomaterials, which included a section dedicated to continuous hydrothermal synthesis as an example of bottom-up synthesis. The review specifically showed that a counter current reactor design had the potential for scaling up, which is the focus of this paper. Continuous hydrothermal synthesis is a relatively new method for the preparation of inorganic nanomaterials, which offers a cheap, green, and highly scalable route $[8,9]$. The process is relatively straightforward; it involves the mixing of an aqueous metal salt stream with a superheated water stream (hydrothermal) within a continuous reactor to produce nano-size particles [10]. If the superheated water reaches its critical point, it changes from a polar liquid to a fluid with a low dielectric constant and low ionic product, which is necessary for some materials [11] but not all [12].

This synthesis method was originally applied to metal oxide-based materials, where it showed potential for the formation of single and complex metal oxides such as $\mathrm{AlOOH}, \mathrm{Fe}_{3} \mathrm{O}_{4}, \mathrm{NiO}, \mathrm{CoFe}_{2} \mathrm{O}_{4}, \mathrm{ZrO}_{2}, \mathrm{CeO}_{2}$, and $\mathrm{TiO}_{2}[8,13]$. However, over the last 10 years, researchers have had considerable success synthesizing a wider range of materials, including metals [14], spinel materials [15], sulfides [16, 17], hydroxides [18, 19], phosphates [20], nitrides [21], and more complex materials such as metal organic frameworks [22]. Figure 1 shows a range of particles from metals to oxides to sulfides that were synthesized during the SHYMAN project, which funded this research work. Non-oxide synthesis has involved the use of a solvothermal method [23], combined hydrothermalsolvothermal method [24], the addition of reductants $[25,26]$, or a selection of reagents to control the oxidation states [27]. Santamaria's review also highlighted the critical need to control the particle size and particle size distribution, and it is this area of quality control that is most affected during the scale up of a manufacturing process from bench to pilot to full scale.

The morphology and size of the particles produced are highly dependent on the mixing mechanism occurring inside the reactor because of its subsequent effect on the nucleation rate of the nanoparticles $[28,29]$. Thus, a suitable design for the mixing device in the supercritical synthesis of nanoparticles is a key parameter for obtaining particles characterized by a small size and narrow particle size distribution (PSD) [10]. Normally, the mixing in these types of devices occurs in a turbulent manner, where turbulent mixing involves the ability of a turbulent flow to effectively mix entrained fluids at a molecular scale [30].

This method can produce nanocrystalline products with mean particle sizes of less than $10 \mathrm{~nm}$ and narrow particle size distributions using a continuous flow reactor [31]. A particularly attractive aspect is that the chemical reactions are usually performed in a single step, which somewhat simplifies the criteria for the reactor design [32].

To obtain a high-throughput and reproducible nanoparticle synthesis technology, the rapid mixing of nanoparticle precursors is required, and the mixing process needs to be highly controlled [33]. Although the influence of mixing on the final shape of the PSD is well known, the search for proxies for PSD shape and particle size prediction has received little attention [34]. Designing a reactor to obtain particles with a narrow PSD (on the nanometer scale) remains challenging because of the uncertainties in the kinetics of supercritical hydrothermal processes. There is a 

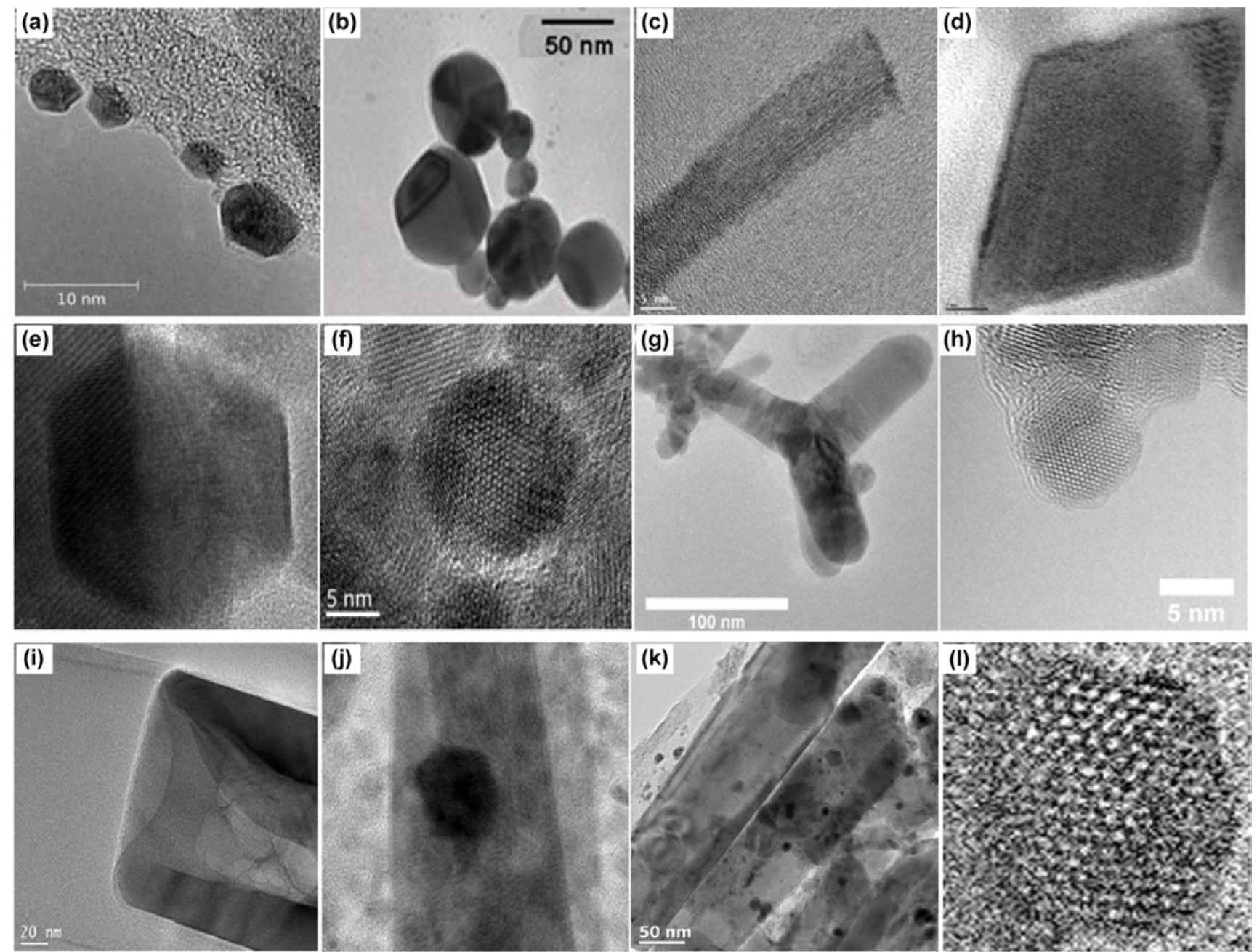

Figure 1 (a) $\mathrm{Au}$, (b) $\mathrm{Ag}$, and (c) $\mathrm{WO}_{3}$, with a $5 \mathrm{~nm}$ scale bar; (d) iron oxide $\left(\mathrm{Fe}_{2} \mathrm{O}_{3}\right.$ ) with a $5 \mathrm{~nm}$ scale bar; (e) $\mathrm{BaTiO}$, with a $2 \mathrm{~nm}$ scale bar; (f) $\mathrm{ZnFe}_{3} \mathrm{O}_{4}$, (g) CdS, (h) $\mathrm{ZnS}$, (i) $\mathrm{Ca}_{10}\left(\mathrm{PO}_{4}\right)_{6}(\mathrm{OH})_{2}$, and (j) Ag inside sheet of $\mathrm{Ca}_{10}\left(\mathrm{PO}_{4}\right)_{6}(\mathrm{OH})_{2}$, with a $5 \mathrm{~nm}$ scale bar; (k) Pt on $\mathrm{TiO}_{2}$ nanotubes and (l) Ni MOF-74, with a $5 \mathrm{~nm}$ scale bar.

relatively limited amount of kinetic data for the wide range of compounds that this technique can produce. Thus, the approximation of PSDs by means of available indicators is highly recommended. Sierra-Pallares et al. reported the equivalence between the PSD and the residence time distribution (RTD) in a reactor during the synthesis of titania nanoparticles in a carbon dioxide medium $[35,36]$. In that work, the PSDs correlated well with the RTDs, and the authors were able to develop a criterion for the optimization of the synthesis, clearly indicating a method for producing a narrower PSD. This criterion was developed in more detail and specifically for continuous hydrothermal synthesis, operating under supercritical synthesis conditions (scHS), in a further publication [37]. In this article, we shall demonstrate how the PSDs can be approached using RTDs as a proxy, and how the RTD can be predicted using computational fluid dynamics (CFD). The synergy between these techniques provides an excellent tool for nanoparticle research. We combine the ideas of chemical reactor engineering and chemical synthesis routes, and propose a new method to deal with the design of the apparatus for scHS.

The aims of the work outlined within this paper are to develop a methodology to experimentally estimate the PSD using the RTD as a proxy, and to use the RTD itself as a validation of the CFD model. In this way, we will be able to use CFD simulations to design a reactor vessel with a new scale with greater 
confidence, and obtain a better understanding of the fluid flow within the system.

\section{Experimental studies}

Experimental data on RTDs were obtained using a nanoparticle synthesis system configuration featuring a counter current mixing reactor, similar to that described by Lester et al., adapted for tracer injection and detection (Fig. 2) [38]. Two high-pressure liquid chromatography (HPLC) pumps were used to deliver water to the reactor. The stream entering the top of the reactor first passed through a custom continuous flow heater, with the flow temperature at the reactor inlet measured by an in-flow thermocouple $\left(T_{1}\right)$; the water flowing into the base of the reactor, which would normally carry a solubilized metal salt during nanoparticle synthesis, was directed via a switching two-position injection valve (Rheodyne 9010 injector), fitted with a $10 \mu \mathrm{L}$ injection loop. The reactor outlet temperature was measured at a second thermocouple $\left(T_{2}\right)$ before the stream was cooled in a heat exchanger, and pressure was relieved at a spring-loaded backpressure regulator (BPR). The switching loop was used to introduce $10 \mu \mathrm{L}$ of tracer solution into the flow entering the base of the reactor, representing the metal salt solution.

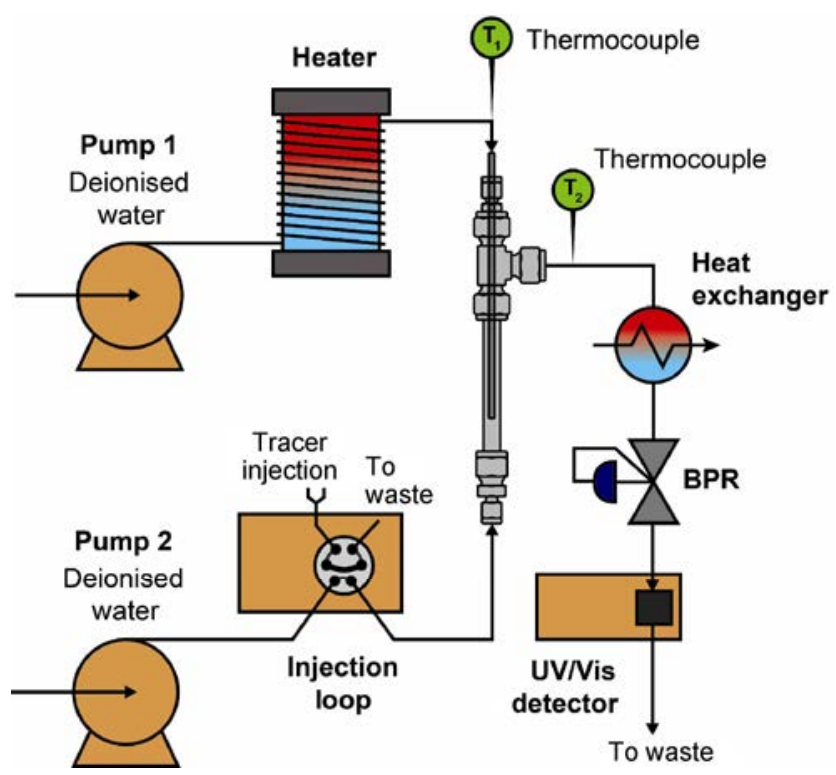

Figure 2 Schematic of system configuration used to inject tracer into reactor and detect it by UV/Vis absorption at system outlet.
After the BPR, the concentration of the tracer eluting from the system was monitored over time using a UV-Vis absorption detector. The resulting absorption trace reflected the distribution of residence times within the system. The distribution results from the flow dynamics included the variable flow paths, flow velocity distributions, flow recycling, and stagnant regions. Aqueous solutions of phenol (Sigma) and nigrosin black (Fischer) at concentrations of $10 \mathrm{~g} \cdot \mathrm{L}^{-1}$ were investigated as tracers, because they were both found to be reasonably stable in supercritical water for the residence times of interest; they were detected using wavelengths of 210 and $545 \mathrm{~nm}$, respectively. Nigrosin black was ultimately used for the studies because, at the highest experimental temperatures, the absorption trace for the phenol tracer (eluting from the system) was seen to vary significantly and erratically between repeat injections (particularly the tail), possibly due to a decrease in solubility under the most extreme conditions, resulting in unpredictable retention.

The acquired UV/Vis absorption traces indicated the distribution of the residence times within the system from the injection point up to the point of detection, but only the measurement of the distribution occurring inside the reactor was required. The additional volumes could not be removed because they were required to cool the flow, maintain pressure, and perform the injection/detection of the tracer. Consequently, a second set of "background" distribution measurements had to be obtained with the reactor removed (while maintaining the same conditions as with the reactor present), which represent the RTD of the system volume excluding the reactor. This background trace was deconvoluted from the trace of the entire system to generate a third trace, which reflected the distribution of the residence times in the reactor volume, as demonstrated in Fig. 3. These deconvoluted RTDs could be directly compared with the CFD model approximations for a given set of process parameters.

The flow rates and (heated) flow temperature were the only parameters varied in this study, because the effect of varying pressure on the residence time was less significant beyond the critical pressure within the permissible range of values. Typically, continuous 


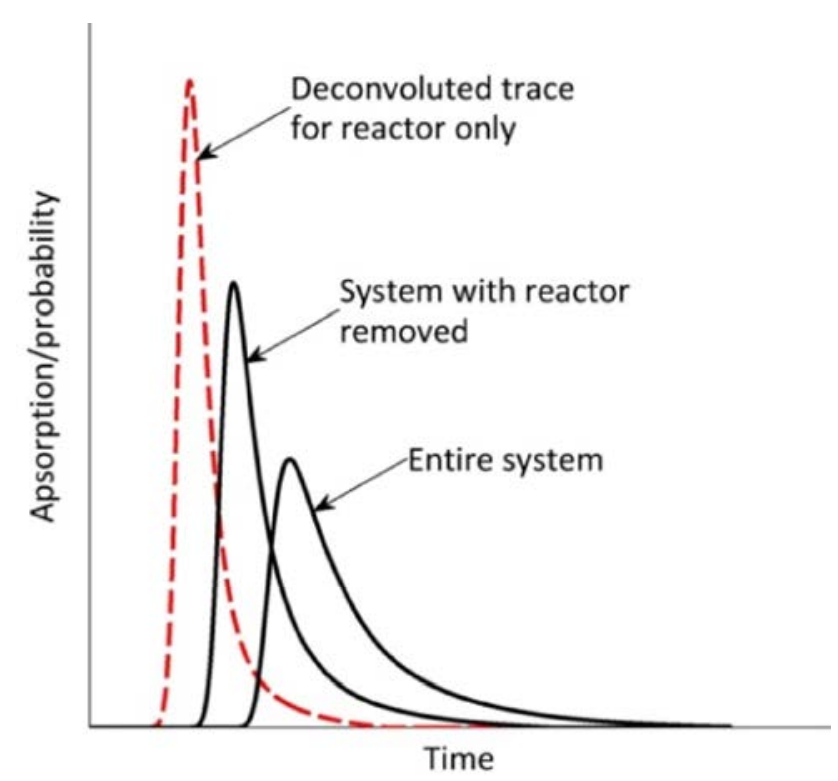

Figure 3 Representative plot illustrating typical absorption traces acquired for two system configurations, and probability distribution for reactor only volume calculated via deconvolution.

supercritical hydrothermal nanoparticle synthesis methods employ a working pressure substantially higher than the critical pressure (22.06 $\mathrm{MPa})$, in order to allow a reasonable margin for the pressure fluctuations resulting from pump pulsations or decreasing BPR efficiency. Thus, a fixed pressure of $25 \mathrm{MPa}$ was employed for the residence time studies. The temperature of the heated stream was varied between ambient and $723.15 \mathrm{~K}$. The typical flow rates employed for nanoparticle synthesis using a counter current reactor are a heated stream flowing at $20 \mathrm{~mL} \cdot \mathrm{min}^{-1}$ downward against an upward flowing ambient temperature metal salt stream at $10 \mathrm{~mL} \cdot \mathrm{min}^{-1}$ (this can be referred to as "20:10", where the heated stream flow rate $\left(\mathrm{mL} \cdot \mathrm{min}^{-1}\right)$ is compared to the metal salt flow rate $\left.\left(\mathrm{mL} \cdot \mathrm{min}^{-1}\right)\right)$. For the work reported in this article, the flow rates employed were 20:20, 20:15, and 20:10. Table 1 lists the details of the simulated experiments, along with the relevant characteristics of the streams involved.

\section{Computational studies}

Numerical simulations of the flow field were conducted using the commercial CFD solver ANSYS Fluent 14.5 in order to solve the nonlinear Navier-Stokes equations, along the realizable $k-\varepsilon$ turbulence model equations, governing the conservation of mass, momentum, and turbulence quantities within the fluid elements. Advection-diffusion equations were also solved to predict the mixing at all scales. The three-dimensional (3D) domain of the hydrothermal reactor (Fig. 4) was meshed using hexahedral finite volumes, with a maximum mesh size of $0.2 \mathrm{~mm}$. The coarsening and refining of the mesh space grid ensured that the solutions were independent of the spatial discretization. The inlet boundary conditions were set for a fully developed velocity profile (calculated on the basis of the flow rate), while a zero-pressure condition was imposed on the outlet, and a non-slip boundary condition was set for the reactor walls. An assumption of symmetry (see the Electronic Supplementary Material (ESM)) allowed the equations to be solved for half of the reactor, reducing the computation time.

A Newtonian fluid was assumed with the properties of water at room temperature and no-slip boundary conditions on all the walls. The mesh independence was verified by comparing results using higher density meshes. The flow rates were specified at the two inlets. The convergence limits were set so that the velocities converged within $0.1 \%$, and the mass fractions for the central stream species reached their asymptotic values within $0.01 \%$.

\section{Results}

\subsection{Model validation}

In order to produce trustworthy results from CFD simulations, thorough validation studies should be conducted. The accuracy of the presented CFD model was assessed through a comparison with the RTDs generated using the previously described tracer methods. A series of calculations was conducted using the CFD model in an attempt to simulate the empirically measured RTDs, imposing the same conditions employed for the experimental work. During this process, three phenomena were explored: the time scale associated with the RTD experiment, uncertainty of the entry signal in the reactor, and unsteadiness of the flow inside the reactor.

The geometry of the reactor is illustrated in Fig. 4. In the experimental setup, the ambient temperature 
Table 1 Experiments performed and simulated

\begin{tabular}{cccccccc}
\hline Case & $\begin{array}{c}Q_{\mathrm{HW}} \\
\left(\mathrm{mL} \cdot \mathrm{min}^{-1}\right)\end{array}$ & $\begin{array}{c}Q_{\mathrm{CW}} \\
\left(\mathrm{mL} \cdot \mathrm{min}^{-1}\right)\end{array}$ & $\begin{array}{c}T_{\mathrm{CW}} \\
(\mathrm{K})\end{array}$ & $\begin{array}{c}T_{\mathrm{HW}} \\
(\mathrm{K})\end{array}$ & $\begin{array}{c}m_{\mathrm{HW}} \\
\left(\mathrm{g} \cdot \mathrm{s}^{-1}\right)\end{array}$ & $\begin{array}{c}m_{\mathrm{CW}} \\
\left(\mathrm{g} \cdot \mathrm{s}^{-1}\right)\end{array}$ & $\begin{array}{c}\text { Reynolds } \\
\mathrm{HW}\end{array}$ \\
\hline 1 & 20 & 10 & 288 & 723.15 & 0.3367 & 0.1683 & 3,044 \\
2 & 20 & 15 & 288 & 723.15 & 0.3367 & 0.2525 & 2,556 \\
3 & 20 & 20 & 288 & 723.15 & 0.3367 & 0.3367 & 2,440 \\
\hline
\end{tabular}

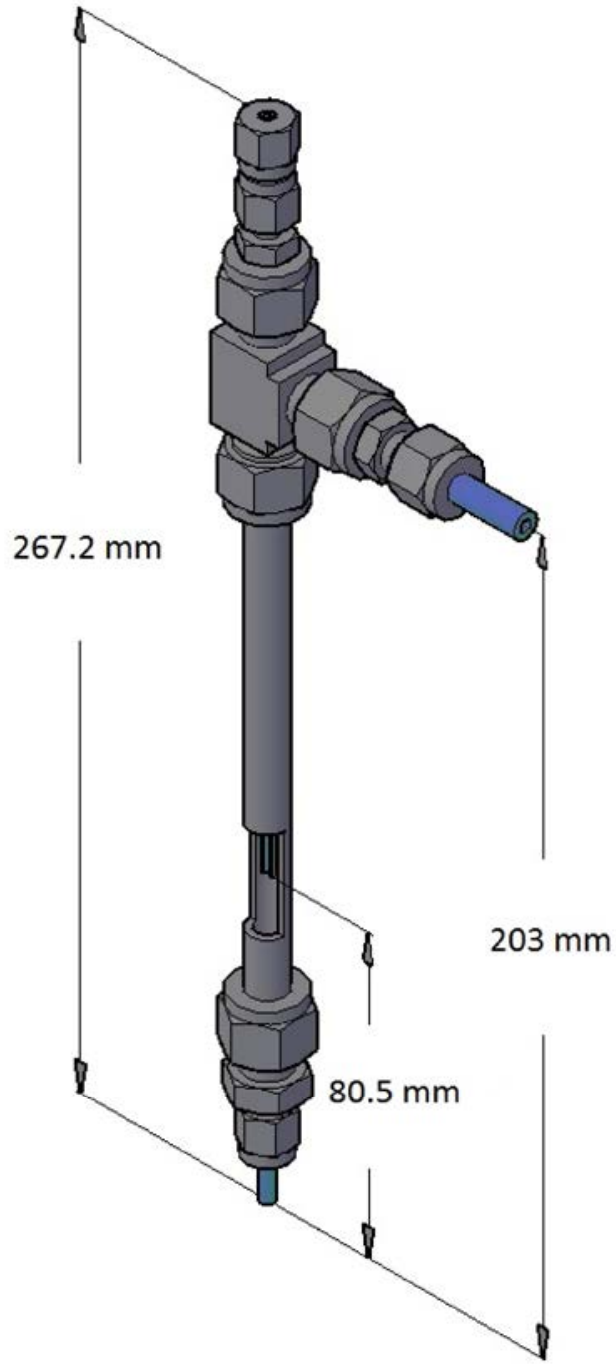

Figure 4 Sketch of the actual reactor with relevant dimensions.

water stream was introduced from the bottom of the reactor, with the heated one at the top. Although, in practice, bent tubing may influence the flow dynamics, we assumed that this effect was negligible. Consequently, the modeled geometry was simpler for the computational setup (see the ESM), making it easier to calculate the flow inside the reactor.
The time scale associated with the empirical RTD experiments was on the same order as that of the mean residence time, i.e., seconds. To adequately model this phenomenon, a significantly smaller time scale was employed for the CFD simulations (around $50 \mathrm{~ms}$ ), in order to eliminate any computational limitations due to the time step.

The flow was carried to the bottom of the reactor via a relatively narrow inlet tube, and although the signal was deconvoluted, modeling the introduction of the flow into the reactor was problematic because of the uncertainties in the composition profile of the tracer. The profile would have been affected by the laminar flow field in the narrow inlet tube, and it was difficult to predict because of the sinuous path of the inlet tube (please see the details in the rig picture of the ESM). To solve this problem, three different signals were tested for the tracer inlet in order to investigate their impact on the final simulated RTD result: a Gaussian profile, step profile, and typical laminar reactor profile [39]. No significant difference was observed between them because of the relative scale of the reactor in comparison to that of the inlet pipe; the reactor effectively diffused the effect of the entry signal. As a result, Gaussian inlet profiles were employed to model the flow of the tracer because they offered computational advantages.

Concerning the unsteadiness of the flow inside the reactor, different simulations were carried out to study its influence on the final shape of the calculated RTD. It is well known from visualization experiments that the flow inside a hydrothermal reactor is unsteady in most cases [40,41]. This phenomenon is the result of natural convection influencing the flow field, as the extreme temperature gradients lead to strong buoyancy forces. Generally speaking, flow fields dominated by buoyancy tend to remain unsteady in nature, forming 
flow structures that evolve in time and space. However, the unsteadiness of the flow was not found to have a significant influence on the computed RTD. Therefore, steady computations were conducted.

The experimentally obtained RTDs and the RTDs predicted by the CFD are depicted in Fig. 5. The prediction of the RTDs using the CFD model appears to very closely match the experimental results. For the lowest flow rates (20:10), an almost perfect match is observed, although some deviations appear at the higher flow rates. Hence, the CFD model appears to be well validated for the range of conditions studied in this work.

The shape of the RTDs is typical of a system dominated by axial dispersion [36]. As previously explained, the tracer was injected into the ambient temperature water flow and transported by convection and diffusion up to the mixing point. At this point, the two streams collided and combined, forming a mixture with an intermediate temperature and density that evolved as it progressed upward through the reactor to the outlet. An important feature of this combined flow was a fairly low mean velocity, with an order of magnitude of centimeters per second. One undesirable effect of this configuration was the fact that the relative influences of the convective transport and turbulent diffusion were comparable in this section of the reactor, which produced a long tail in the RTD. This effect was distinctly observed in both the simulated and experimental RTD results.

Additionally, a dead volume was found toward the top of the reactor, which would also have contributed to the long tail. Both effects will be studied using the validated $C F D$ model.

Figure 6 shows the temperature distribution and internal residence time distribution computed with the CFD model (please see the ESM for details on the equations solved). These plots present an explanation of the flow features associated with the RTD signals. The temperature gradient along the reactor is seen to be concentrated in two different regions: the mixing point and upper region of the reactor (region $\mathrm{H} 3$ in Fig. 6). At the mixing point, excellent macro-scale mixing of the two streams is observed, affording complete temperature homogenization in an extremely short space. The hot water stream conserves much of its enthalpy as it jets downward from the outlet of the inner pipe for a distance approximately equivalent to two to three times the inner pipe's inner diameter, and then cools down to the final mixing temperature very rapidly. The combined flow travelling through the annulus is very uniform from plane $\mathrm{H} 1$ up to plane H2 (see Fig. 6). However, in the top section of the reactor (plane $\mathrm{H} 3$ ), there is a potentially undesirable flow structure. This region is much hotter that in the annulus of the reactor, and the mean velocity is small (stagnant flow) because it is not located in the main pathway through the outlet. Thus, it is highly probable that this region may facilitate the uncontrolled growth of nanoparticles.

This last statement is demonstrated by the internal residence time distribution depicted in Fig. 6 (right), which was computed using the CFD. The contour plot is saturated to visually highlight the effect, where
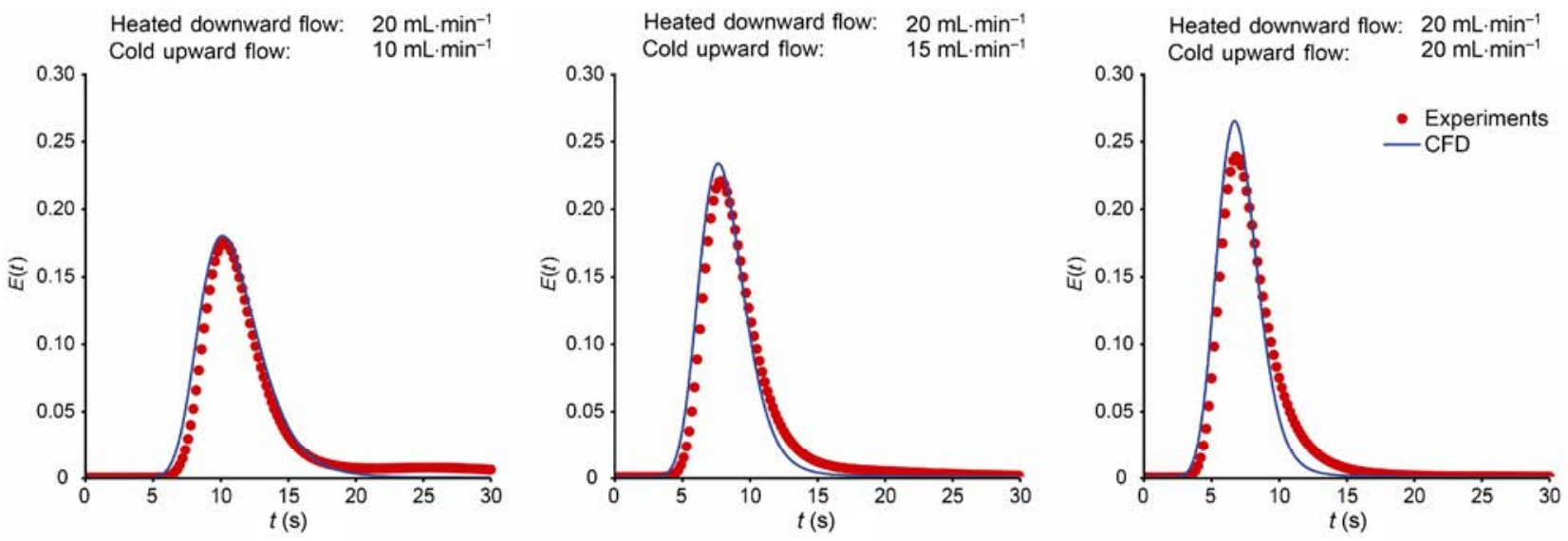

Figure 5 Experimental and predicted RTDs (see Table 1). 

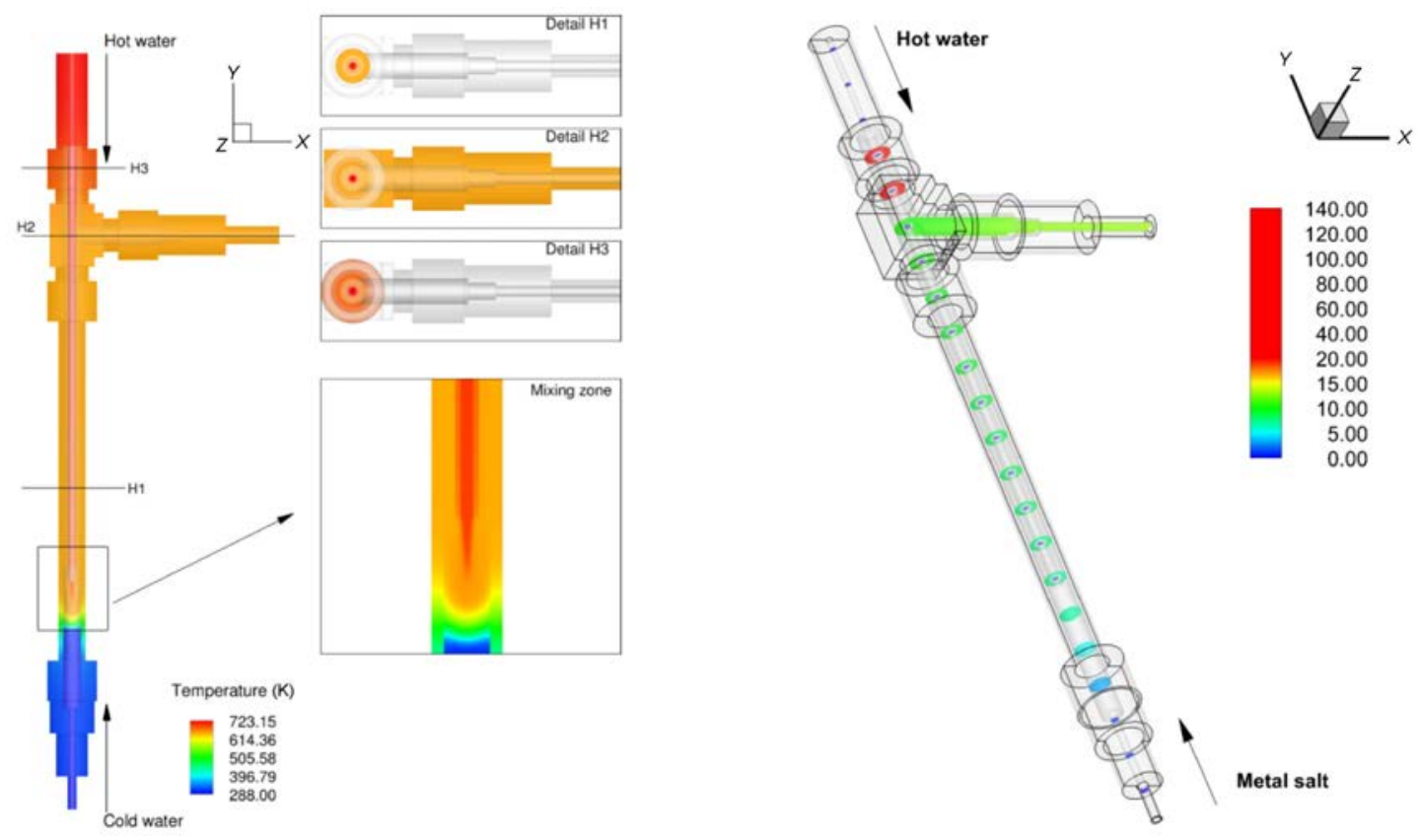

Figure 6 Temperature distribution in symmetry plane of reactor (left) and internal residence time distribution (right) in different slices from ambient temperature inlet to reactor outlet, indicating dead volume toward the top of reactor. The results correspond to case 1 (20:10).

the red color indicates a residence time greater than the mean residence time $(9.32 \mathrm{~s})$. This residence time distribution corresponds to the flow of cold water. The residence time is seen to increase along the main pathway through the reactor geometry up to the exit; however, as predicted, there is a significantly extended residence time located in the top section. Therefore, the model was demonstrated to be highly efficient in the interpretation of the shape of the RTD data and made it possible to identify the potential undesirable regions.

\subsection{Equivalence between RTD shapes and PSD shapes}

A remarkable aspect of this research was the equivalence found between the shape of the RTD data and the shape of the experimental PSDs. Figure 7 shows this equivalence for cases 1-3 and highlights the observed trend. See the ESM for more information about the HRTEM imaging.

Such equivalence has been reported for other systems in the prior literature, with some authors speculating about the importance of the RTD in relation to the PSD. However, to the best of our knowledge, this is the first time that the relationship has been experimentally confirmed for hydrothermal synthesis $[35,37]$. As illustrated in Fig. 7, the trend for the changing shape of the PSDs closely matches that of the RTDs. This is because the particles closely follow the fluid flow, and the residence time has a direct impact on the particle growth and resultant size. In this way, particles drifting away from the primary flow paths through the reactor will have higher residence times and therefore larger final particle diameters. Thus, to obtain narrow PSDs and small nanoparticles, it is crucial to accurately control the RTD. This result has direct implications when considering the design of equipment beyond the bench or pilot scale toward full-scale nanoparticle production.

\subsection{Flow regimes and mixing time scales}

The CFD code was also useful for determining the regions of the reactor where mixing occurred at different space scales. The macro-scale was studied through an analysis of the temperature distribution, as shown in Fig. 6. Additionally, a mixing model was employed to explore mixing at the micro-scale in the reactor. Figure 8 shows a contour plot of the segregation 

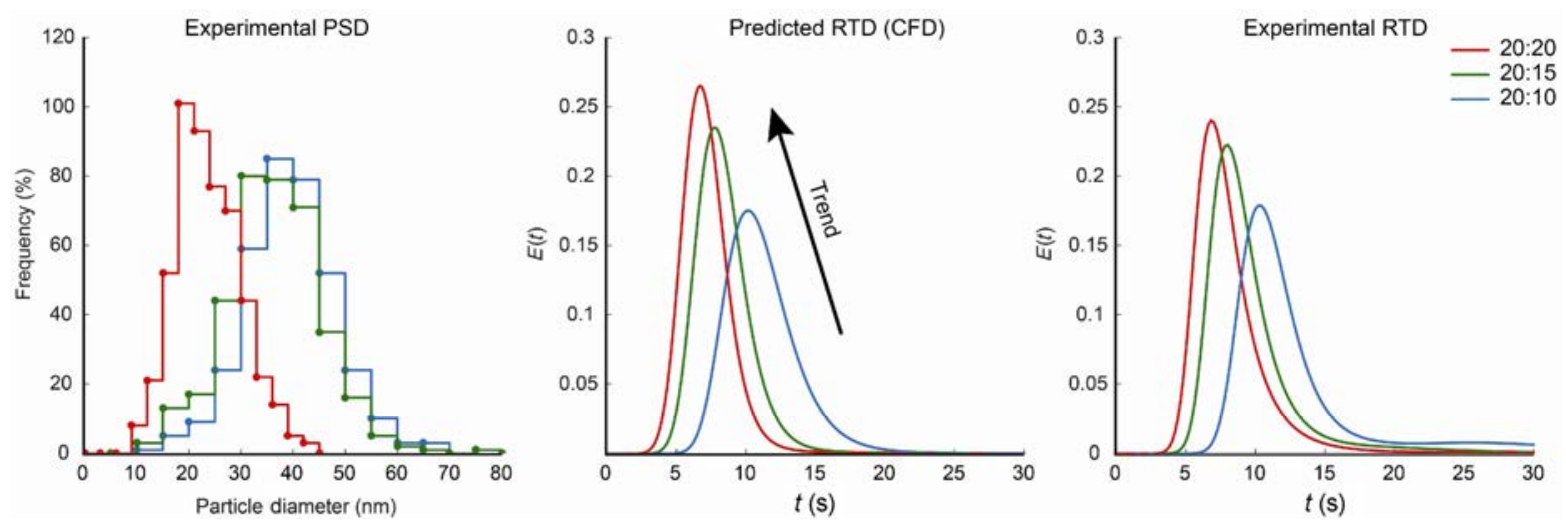

Figure 7 Experimental PSDs obtained for production of hematite, along with RTDs experimentally measured and predicted RTDs using the CFD code.

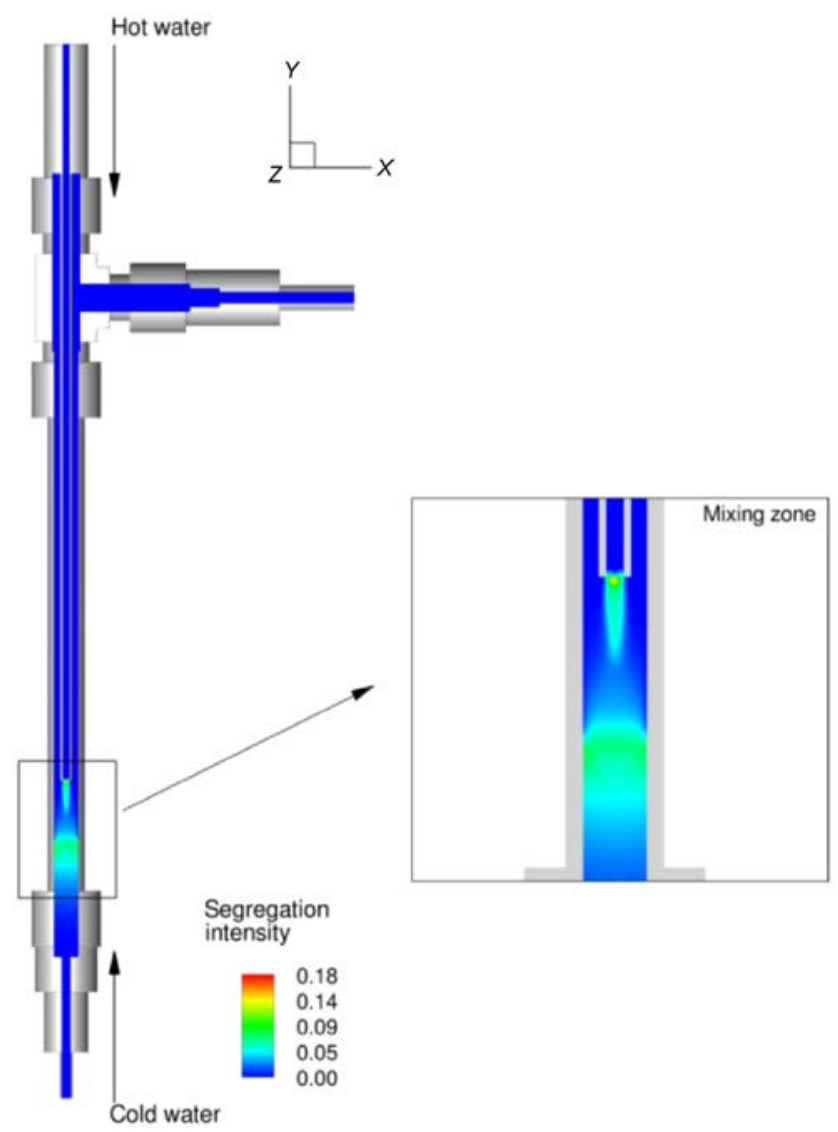

Figure 8 Segregation intensity found for case 1, indicating regions where molecular mixing takes place.

intensity [42]. The segregation intensity is a measure of mixing at the macro- and micro-scales, and it is defined as the extent to which the tracer concentration departs from the mean [43]. If the purpose of mixing two fluids A and B is to enable them to react with one another, the segregation intensity represents a measure of the effectiveness of the mixing at all scales. An analysis of the segregation intensity pattern in Fig. 8 reveals an interesting behavior close to the mixing point. A region located just below the jet appears to accommodate substantial micro-scale mixing, with a mixing performance somewhat similar to that of the jetting zone. The main difference compared to the jetting region is that there is no macro-scale mixing (it is undetectable in the temperature distribution), but mixing occurs only at the micro-scale owing to the turbulent diffusion below the jet. As a consequence, this zone may significantly influence nanoparticle production, because chemical reactions occur at the molecular level. The detection of this region was only possible using the validated CFD code with an adequate mixing model.

\section{Conclusions}

The results of experimental tracer studies under reaction conditions typically employed for the synthesis of nanomaterials showed a strong correlation to CFD simulations that predicted the residence time and PSDs. The size distribution data empirically derived from the counter current scHS reactor validated the CFD model.

This work demonstrated an effective method for reactor or system profiling using tracer technology, where deconvolution techniques were able to successfully resolve the RTDs for a specific part of the continuous flow apparatus. 
The potential application of CFD modeling to continuous supercritical flow reactors was also demonstrated, showing the ability to profile the dominant mixing regimes within different regions of the reactor and investigate the mixing on different scales.

\section{Acknowledgements}

This work is funded by the European Union's Seventh Framework Programme (FP7/2007-2013), grant agreement no. FP7-NMP4-LA-2012-280983, SHYMAN.

\section{Electronic Supplementary Material: Supplementary} material (the modelling and empirical measurement of the nanoparticles) is available in the online version of this article at http://dx.doi.org/10.1007/s12274-0161215-6.

\section{References}

[1] Hobson, D. W. Commercialization of nanotechnology. Wiley Interdiscip. Rev. Nanomed. Nanobiotechnol. 2009, 1, 189-202.

[2] Seo, Y. H.; Jeong, S.; Jo, Y.; Choi, Y.; Ryu, B.-H.; Han, G.; Lee, M. Long-term dispersion stability and adhesion promotion of aqueous $\mathrm{Cu}$ nano-ink for flexible printed electronics. $J$. Nanosci. Nanotechnol. 2013, 13, 5661-5664.

[3] Syamchand, S. S.; Sony, G. Europium enabled luminescent nanoparticles for biomedical applications. J. Lumin. 2015, 165, 190-215.

[4] Uludag, Y.; Köktürk, G. Determination of prostate-specific antigen in serum samples using gold nanoparticle based amplification and lab-on-a-chip based amperometric detection. Microchim. Acta 2015, 182, 1685-1691.

[5] Middlemas, S.; Fang, Z. Z.; Fan, P. Life cycle assessment comparison of emerging and traditional titanium dioxide manufacturing processes. J. Clean. Prod. 2015, 89, 137-147.

[6] Zhang, Y.; Zhang, L. Y.; Zhou, C. W. Review of chemical vapor deposition of graphene and related applications. Acc. Chem. Res. 2013, 46, 2329-2339.

[7] Sebastian, V.; Arruebo, M.; Santamaria, J. Reaction engineering strategies for the production of inorganic nanomaterials. Small 2014, 10, 835-853.

[8] Byrappa, K.; Adschiri, T. Hydrothermal technology for nanotechnology. Prog. Cryst. Growth Ch. 2007, 53, 117-166.

[9] Adschiri, T.; Kanazawa, K.; Arai, K. Rapid and continuous hydrothermal crystallization of metal oxide particles in supercritical water. J. Am. Ceram. Soc. 1992, 75, 1019-1022.

[10] Lester, E.; Blood, P.; Denyer, J.; Giddings, D.; Azzopardi, B.; Poliakoff, M. Reaction engineering: The supercritical water hydrothermal synthesis of nano-particles. J. Supercrit. Fluid. 2006, 37, 209-214.

[11] Adschiri, T.; Hakuta, Y.; Sue, K.; Arai, K. Hydrothermal synthesis of metal oxide nanoparticles at supercritical conditions. J. Nanopart. Res. 2001, 3, 227-235.

[12] Aoki, N.; Sato, A.; Sasaki, H.; Litwinowicz, A.-A.; Seong, G.; Aida, T.; Hojo, D.; Takami, S.; Adschiri, T. Kinetics study to identify reaction-controlled conditions for supercritical hydrothermal nanoparticle synthesis with flow-type reactors. J. Supercrit. Fluid. 2016, 110, 161-166.

[13] Byrappa, K.; Ohara, S.; Adschiri, T. Nanoparticles synthesis using supercritical fluid technology-Towards biomedical applications. Adv. Drug Deliv. Rev. 2008, 60, 299-327.

[14] Aksomaityte, G.; Poliakoff, M.; Lester, E. The production and formulation of silver nanoparticles using continuous hydrothermal synthesis. Chem. Eng. Sci. 2013, 85, 2-10.

[15] Nugroho, A.; Yoon, D.; Chung, K. Y.; Kim, J. Synthesis of $\mathrm{Li}_{4} \mathrm{Ti}_{5} \mathrm{O}_{12}$ /carbon nanocomposites in supercritical methanol for anode in Li-ion batteries: Effect of surface modifiers. J. Supercrit. Fluid. 2015, 101, 72-80.

[16] Dunne, P. W.; Munn, A. S.; Starkey, C. L.; Lester, E. H. The sequential continuous-flow hydrothermal synthesis of molybdenum disulphide. Chem. Commun. 2015, 51, 4048-4050.

[17] Dunne, P. W.; Starkey, C. L.; Gimeno-Fabra, M.; Lester, E. H. The rapid size- and shape-controlled continuous hydrothermal synthesis of metal sulphide nanomaterials. Nanoscale 2014, 6, 2406-2418.

[18] Adschiri, T.; Takami, S.; Arita, T.; Hojo, D.; Minami, K.; Aoki, N.; Togashi, T. Supercritical hydrothermal synthesis. In Handbook of Advanced Ceramics, $2^{\text {nd }}$ ed.; Somiya, S., Ed.; Academic Press: Oxford, 2013; pp 949-978.

[19] Wang, Q.; Tang, S. V. T.; Lester, E.; O’Hare, D. Synthesis of ultrafine layered double hydroxide (LDHs) nanoplates using a continuous-flow hydrothermal reactor. Nanoscale 2013, 5, 114-117.

[20] Chaudhry, A. A.; Haque, S.; Kellici, S.; Boldrin, P.; Rehman, I.; Khalid, F. A.; Darr, J. A. Instant nano-hydroxyapatite: A continuous and rapid hydrothermal synthesis. Chem. Commun. 2006, 2286-2288.

[21] Giroire, B.; Marre, S.; Garcia, A.; Cardinal, T.; Aymonier, C. Continuous supercritical route for quantum-confined GaN nanoparticles. React. Chem. Eng. 2016, 1, 151-155.

[22] Gimeno-Fabra, M.; Munn, A. S.; Stevens, L. A.; Drage, T. C.; Grant, D. M.; Kashtiban, R. J.; Sloan, J.; Lester, E.; Walton, 
R. I. Instant MOFs: Continuous synthesis of metal-organic frameworks by rapid solvent mixing. Chem. Commun. 2012, 48, 10642-10644.

[23] Nugroho, A.; Veriansyah, B.; Kim, S. K.; Lee, B. G.; Kim, J.; Lee, Y. W. Continuous synthesis of surface-modified nanoparticles in supercritical methanol: A facile approach to control dispersibility. Chem. Eng. J. 2012, 193-194, 146-153.

[24] Munn, A. S.; Dunne, P. W.; Tang, S. V. Y.; Lester, E. H. Large-scale continuous hydrothermal production and activation of ZIF-8. Chem. Commun. 2015, 51, 12811-12814.

[25] Seong, G.; Adschiri, T. The reductive supercritical hydrothermal process, a novel synthesis method for cobalt nanoparticles: Synthesis and investigation on the reaction mechanism. Dalton Trans. 2014, 43, 10778-10786.

[26] Arita, T.; Hitaka, H.; Minami, K.; Naka, T.; Adschiri, T. Synthesis of iron nanoparticle: Challenge to determine the limit of hydrogen reduction in supercritical water. J. Supercrit. Fluid. 2011, 57, 183-189.

[27] Seong, G.; Takami, S.; Arita, T.; Minami, K.; Hojo, D.; Yavari, A. R.; Adschiri, T. Supercritical hydrothermal synthesis of metallic cobalt nanoparticles and its thermodynamic analysis. J. Supercrit. Fluid. 2011, 60, 113-120.

[28] Blood, P. J.; Denyer, J. P.; Azzopardi, B. J.; Poliakoff, M.; Lester, E. A versatile flow visualisation technique for quantifying mixing in a binary system: Application to continuous supercritical water hydrothermal synthesis (SWHS). Chem. Eng. Sci. 2004, 59, 2853-2861.

[29] Sugioka, K.; Ozawa, K.; Kubo, M.; Tsukada, T.; Takami, S.; Adschiri, T.; Sugimoto, K.; Takenaka, N.; Saito, Y. Relationship between size distribution of synthesized nanoparticles and flow and thermal fields in a flow-type reactor for supercritical hydrothermal synthesis. J. Supercrit. Fluid. 2016, 109, 43-50.

[30] Dimotakis, P. E. Turbulent mixing. Annu. Rev. Fluid Mech. 2005, 37, 329-356.

[31] Becker, J.; Hald, P.; Bremholm, M.; Pedersen, J. S.; Chevallier, J.; Iversen, S. B.; Iversen, B. B. Critical size of crystalline $\mathrm{ZrO}_{2}$ nanoparticles synthesized in near- and supercritical water and supercritical isopropyl alcohol. ACS Nano 2008, 2, 1058-1068.

[32] Cabañas, A.; Darr, J. A.; Lester, E.; Poliakoff, M. Continuous hydrothermal synthesis of inorganic materials in a near-critical water flow reactor; the one-step synthesis of nano-particulate $\mathrm{Ce}_{1-x} \mathrm{Zr}_{x} \mathrm{O}_{2}(x=0-1)$ solid solutions. J. Mater. Chem. 2001, 11, 561-568.

[33] Lim, J.-M.; Swami, A.; Gilson, L. M.; Chopra, S.; Choi, S.; Wu, J.; Langer, R.; Karnik, R.; Farokhzad, O. C. Ultra-high throughput synthesis of nanoparticles with homogeneous size distribution using a coaxial turbulent jet mixer. ACS Nano 2014, 8, 6056-6065.

[34] Lester, E.; Blood, P. J.; Denyer, J. P.; Azzopardi, B. J.; Li, J.; Poliakoff, M. Impact of reactor geometry on continuous hydrothermal synthesis mixing. Mater. Res. Innov. 2010, 14, 19-26.

[35] Sierra-Pallares, J.; Alonso, E.; Montequi, I.; Cocero, M. J. Particle diameter prediction in supercritical nanoparticle synthesis using three-dimensional CFD simulations. Validation for anatase titanium dioxide production. Chem. Eng. Sci. 2009, 64, 3051-3059.

[36] Levenspiel, O. Tracer Technology: Modeling the Flow of Fluids; Springer: New York, 2012.

[37] Sierra-Pallares, J.; Marchisio, D. L.; Alonso, E.; ParraSantos, M. T.; Castro, F.; Cocero, M. J. Quantification of mixing efficiency in turbulent supercritical water hydrothermal reactors. Chem. Eng. Sci. 2011, 66, 1576-1589.

[38] Cabanas, A.; Darr, J. A.; Lester, E.; Poliakoff, M. A continuous and clean one-step synthesis of nano-particulate $\mathrm{Ce}_{1-x} \mathrm{Zr}_{x} \mathrm{O}_{2}$ solid solutions in near-critical water. Chem. Commun. 2000, 901-902.

[39] Fogler, H. S. Essentials of Chemical Reaction Engineering; Pearson Education: Boston, 2010.

[40] Lester, E.; Blood, P.; Denyer, J.; Giddings, D.; Azzopardi, B.; Poliakoff, M. Reaction engineering: The supercritical water hydrothermal synthesis of nano-particles. J. Supercrit. Fluid. 2006, 37, 209-214.

[41] Aizawa, T.; Masuda, Y.; Minami, K.; Kanakubo, M.; Nanjo, H.; Smith, R. L. Direct observation of channel-tee mixing of high-temperature and high-pressure water. J. Supercrit. Fluid. 2007, 43, 222-227.

[42] Liu, Y.; Fox, R. O. CFD predictions for chemical processing in a confined impinging-jets reactor. AIChE J. 2006, 52, 731-744.

[43] Danckwerts, P. V. The definition and measurement of some characteristics of mixtures. Appl. Sci. Res. A 1952, 3, 279-296. 



\section{Table of contents}





\section{Electronic Supplementary Material}

\section{Understanding bottom-up continuous hydrothermal synthesis of nanoparticles using empirical measurement and computational simulation}

José Sierra-Pallares ${ }^{1}$, Thomas Huddle ${ }^{2}$, Juan García-Serna ${ }^{3}$, Esther Alonso ${ }^{3}$, Fidel Mato ${ }^{3}$, Igor Shvets ${ }^{4}$, Olaf Luebben ${ }^{4}$, María José Cocero ${ }^{3}$, and Edward Lester ${ }^{2}(\square)$

' Fluids Engineering Group Energy and Fluid Mechanics Engineering Department, University of Valladolid, Paseo del Cauce 59, 47010 Valladolid, Spain

${ }^{2}$ Advanced Materials Research Group, the University of Nottingham, University Park, Nottingham NG7 2RD, UK

${ }^{3}$ High Pressure Process Group. C/Prado de la Magdalena s/n, Department of Chemical Engineering and Environmental Technology, University of Valladolid, 47011 Valladolid, Spain

${ }^{4}$ School of Physics, Trinity College Dublin, College green, Dublin 2, Ireland

Supporting information to DOI 10.1007/s12274-016-1215-6

\section{S1 Reactor geometry modelling}

Figure S1(a) details relevant dimensions of the counter current reactor configured for the experimental tracer RTD measurement experiments. In order to produce a workable computational model the geometry was simplified to the configuration indicated in Fig. S1(b). This simplified arrangement made it much easier to compute flow within the reactor. The cross-sectional images in Fig. S1(b) also illustrate the mesh employed in the computations. The number of cells has been carefully analyzed and optimized for size, minimizing computational expense while assuring the results are mesh-independent.

Address correspondence to Edward.Lester@nottingham.ac.uk 
(a)

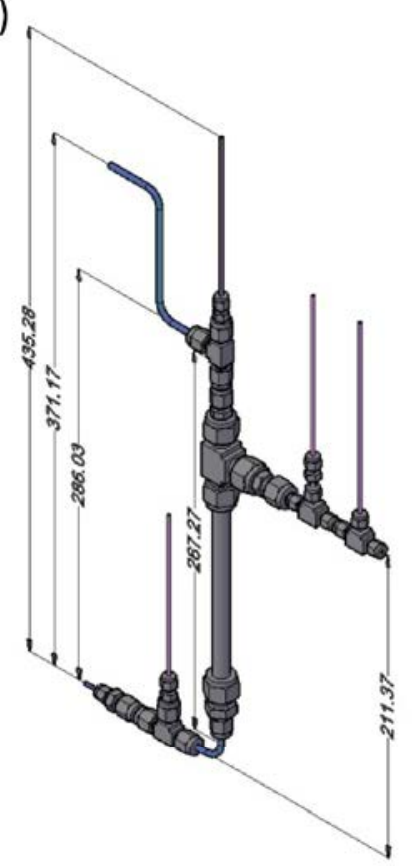

(b)

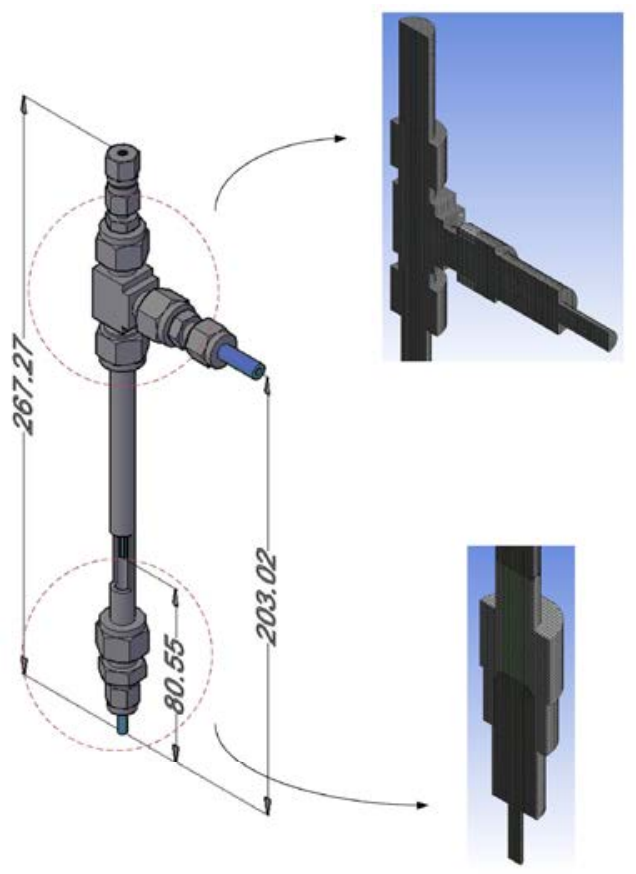

Figure S1 Reactor dimensions along with details of the mesh employed. Dimensions are in millimeters.

\section{S2 System of equations being solved}

The flow and mixing of fluids under supercritical conditions are governed by fundamental equations for conservation of mass, momentum and energy [S1]. When dealing with turbulent flow, a suitable averaging method must be used. The density averaging technique, or Favre averaging, applies fluctuation to average values in lieu of actual flow parameter in order to derive a time averaged solution. The density averaged conservation equations for momentum, mass and energy are represented as

$$
\begin{gathered}
\frac{\partial \bar{\rho}}{\partial t}+\frac{\partial \bar{\rho} \tilde{u}_{j}}{\partial x_{j}}=0 \\
\frac{\partial \bar{\rho} \tilde{u}_{i}}{\partial t}+\frac{\partial}{\partial x_{j}}\left(\bar{\rho} \tilde{u}_{j} \tilde{u}_{i}\right)=-\frac{\partial \tilde{p}}{\partial x_{i}}+\frac{\partial}{\partial x_{j}}\left(\tilde{\sigma}_{i j}-\bar{\rho} \widetilde{u_{l}^{\prime \prime} u_{J}^{\prime \prime}}\right)+\rho g_{i} \\
\frac{\partial \bar{\rho} \tilde{e}}{\partial t}+\frac{\partial}{\partial x_{j}}\left(\bar{\rho} \tilde{u}_{j}\left[\tilde{e}+\frac{\tilde{p}}{\bar{\rho}}\right]\right)=\frac{\partial}{\partial x_{j}}\left[\lambda \frac{\partial \tilde{T}}{\partial x_{j}}+\tilde{u}_{j}\left(\tilde{\sigma}_{i j}-\widetilde{\rho} \widetilde{u_{l}^{\prime \prime} u_{J}^{\prime \prime}}\right)-\bar{\rho} \widetilde{e^{\prime \prime} u_{J}^{\prime \prime}}\right] \\
\frac{\partial \bar{\rho} \tilde{\phi}}{\partial t}+\frac{\partial}{\partial x_{j}}\left(\bar{\rho} \tilde{u}_{j} \tilde{\phi}\right)=\frac{\partial}{\partial x_{j}}\left[\bar{\rho} \mathcal{D} \frac{\partial \tilde{\phi}}{\partial x_{j}}-\bar{\rho} \widetilde{\phi^{\prime \prime} u_{J}^{\prime \prime}}\right]
\end{gathered}
$$

where $u_{j}$ is the velocity component in the $x_{j}$ direction; $p$ is the pressure; $\rho$ is the density; $e$ is the internal energy; $\phi$ is a passive scalar; $\sigma_{i j}$ is the viscous stress tensor and $g_{i}$ is the gravitational acceleration in the $x_{i}$ direction. Neglecting viscosity fluctuations, $\tilde{\sigma}_{i j}$ can be rearranged to

$$
\sigma_{i j}=\eta\left[\left(\frac{\partial \tilde{u}_{i}}{\partial x_{j}}+\frac{\partial \tilde{u}_{j}}{\partial x_{i}}\right)-\frac{2}{3} \delta_{i j} \frac{\partial \tilde{u}_{k}}{\partial x_{k}}\right]
$$


where $\eta$ is the molecular viscosity. Equations (S1)-(S3) are unclosed, because the averaging procedure creates new variables for the Reynolds stress tensor, $-\bar{\rho} \widehat{u_{l}^{\prime \prime} u_{J}^{\prime \prime}}$. To consolidate interdependent variables and reduce the number of unknowns, turbulent closure must be employed. There are different approaches to predicting turbulent flows ranging from Reynolds-averaged Navier-Stokes (RANS) to large eddy simulation (LES) and direct numerical simulation (DNS). In this paper RANS models are used, where the primary task is to model the Reynolds stress tensor. Generally, it is calculated using the Boussinesq hypothesis, which requires calculation of the turbulent viscosity $\mu^{\mathrm{T}}$ and turbulence kinetic energy $k$.

$$
-\bar{\rho} \widetilde{u_{l}^{\prime \prime} u_{J}^{\prime \prime}}=\rho \frac{2}{3} \delta_{i j} k-\eta^{\mathrm{T}}\left(\frac{\partial \tilde{u}_{i}}{\partial x_{j}}+\frac{\partial \tilde{u}_{j}}{\partial x_{i}}\right)
$$

with $k=\frac{1}{2} \widetilde{u_{l}^{\prime \prime} u_{J}^{\prime \prime}}$. Similarly,

$$
-\bar{\rho} \widetilde{e^{\prime \prime} u_{J}^{\prime \prime}}=\lambda^{\mathrm{T}} \frac{\partial \tilde{T}}{\partial x_{j}}, \quad-\bar{\rho} \widehat{\phi^{\prime \prime} u_{J}^{\prime \prime}}=\mathcal{D}^{\mathrm{T}} \frac{\partial \tilde{\phi}}{\partial x_{j}}
$$

where $\lambda^{\mathrm{T}}$ and $D^{\mathrm{T}}$ are the turbulent conductivity and viscosity respectively. Both quantities are computed from the turbulent viscosity using the relationships

$$
\lambda^{\mathrm{T}}=\frac{C_{P} \eta^{\mathrm{T}}}{\operatorname{Pr}^{\mathrm{T}}} \quad \mathcal{D}^{\mathrm{T}}=\frac{\eta^{\mathrm{T}}}{\bar{\rho} \mathrm{Sc}^{\mathrm{T}}}
$$

where $\operatorname{Pr}^{T}$ and $\mathrm{Sc}^{\mathrm{T}}$ are the turbulent Prandtl and Schmidt numbers respectively. Both values are normally constant in a turbulent computation, however, for transcritical injection of water, a correlation with the molecular Prandtl number has been recommended by Schuler et al. [S2]. Consequently, turbulent closure requires the use of a model for the turbulent viscosity. In this work, the Realizable $k-\varepsilon$ model of Shi et al. [S3] has been employed for undertaking this task.

Macro and micromixing are analyzed by means of the mixture fraction $(\xi)$ and mixture fraction variance $\left(\xi^{\prime 2}\right)$ equations. Both are treated as passive scalars without molecular diffusivity. The governing equations are respectively

$$
\begin{gathered}
\frac{\partial \bar{\rho} \tilde{\xi}}{\partial t}+\frac{\partial}{\partial x_{j}}\left(\bar{\rho} \tilde{u}_{j} \tilde{\xi}\right)=\frac{\partial}{\partial x_{j}}\left[\bar{\rho} \mathcal{D}^{\mathrm{T}} \frac{\partial \tilde{\xi}}{\partial x_{j}}\right] \\
\frac{\partial \bar{\rho} \widetilde{\xi^{\prime 2}}}{\partial t}+\frac{\partial}{\partial x_{j}}\left(\bar{\rho} \tilde{u}_{j} \widetilde{\xi^{\prime 2}}\right)=\frac{\partial}{\partial x_{j}}\left[\bar{\rho} \mathcal{D}^{\mathrm{T}} \frac{\partial \widetilde{\xi^{\prime 2}}}{\partial x_{j}}\right]+2 \bar{\rho} \mathcal{D}^{\mathrm{T}} \frac{\partial \tilde{\xi}}{\partial x_{j}} \frac{\partial \tilde{\xi}}{\partial x_{j}}-2 \bar{\rho} \gamma \widetilde{\xi^{\prime 2}}
\end{gathered}
$$

where $\gamma$ is the micromixing velocity, calculated according to [S4].

The internal residence time $(\tau)$ is computed using the equation obtained by Ghirelli et al. [S4]

$$
\frac{\partial \bar{\rho} \widetilde{\tau \xi}}{\partial t}+\frac{\partial}{\partial x_{j}}\left(\bar{\rho} \tilde{u}_{j} \widetilde{\tau \xi}\right)=\frac{\partial}{\partial x_{j}}\left[\bar{\rho} \mathcal{D}^{\mathrm{T}} \frac{\partial \widetilde{\tau \xi}}{\partial x_{j}}\right]+\bar{\rho} \tilde{\xi}
$$

\section{S3 Imaging of particles from empirical experiments}

The imaging of the particles was carried out using a FEI Titan TEM at Trinity College Dublin. At least 20 images at a magnification of $69,000 \mathrm{X}$ were captured for each sample. Each image was printed and the scaling 
for each image was then used to manually size the images in a similar way to other publications [S5] using the open source software ImageJ version 1.49k. Figure S3 shows examples of the HRTEM images.

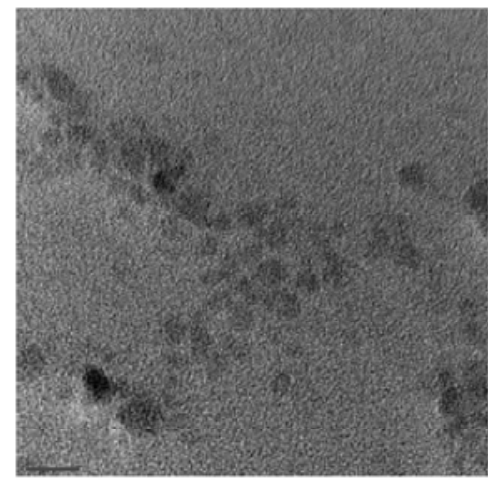

$300{ }^{\circ} \mathrm{C}$

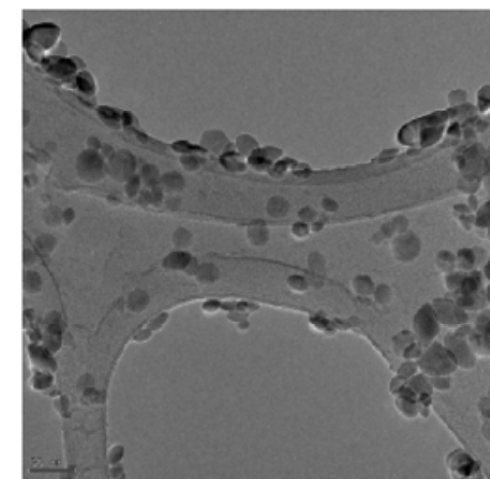

$400{ }^{\circ} \mathrm{C}$

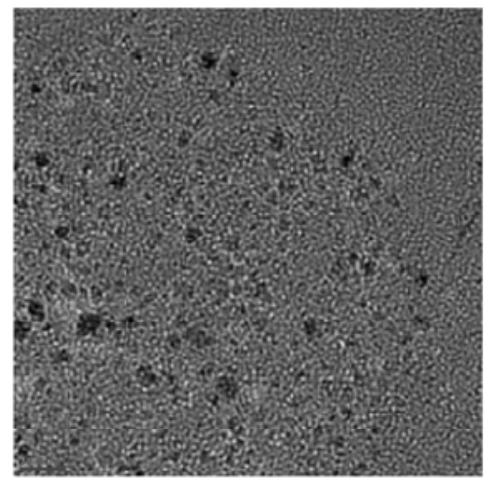

20:15

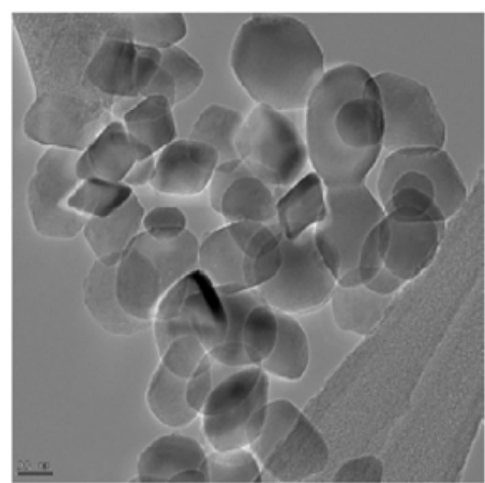

20:15

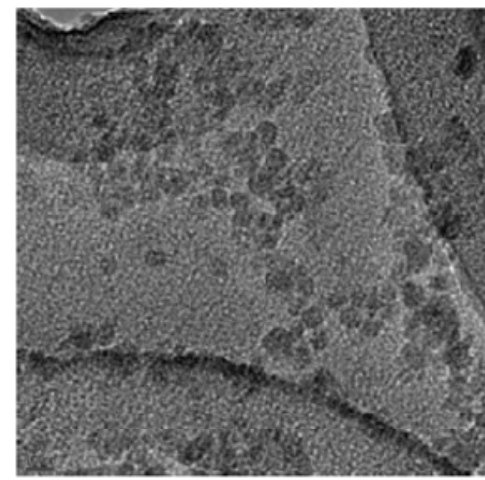

20:10

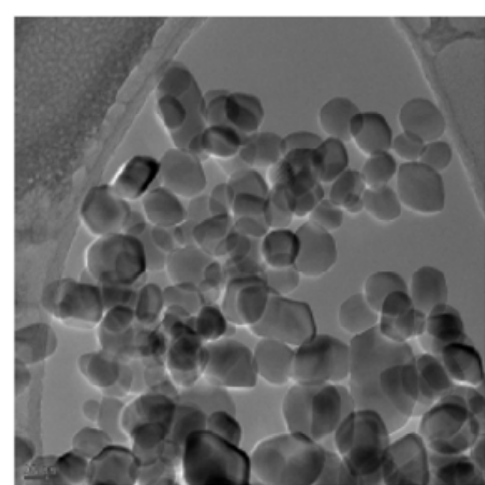

$20: 10$

Figure S3 High resolution transmission electron microscope images of the iron oxide particles produced at 300 and $400{ }^{\circ} \mathrm{C}$ at normal flow conditions.

\section{References}

[S1] Bird, R. B.; Stewart, W. E.; Lightfoot, E. N. Transport Phenomena; Wiley: New York, 2007.

[S2] Schuler, M. J.; Rothenfluh, T.; Stathopoulos, P.; Brkic, D.; Meier, T.; von Rohr, P. R. Simulating supercritical water jets with a variable turbulent Prandtl number. Chem. Eng. Technol. 2014, 37, 1896-1902.

[S3] Shih, T.-H.; Liou, W. W.; Shabbir, A.; Yang, Z. G.; Zhu, J. A new k- $\epsilon$ eddy viscosity model for high Reynolds number turbulent flows. Comput. Fluids 1995, 24, 227-238.

[S4] Ghirelli, F.; Leckner, B. Transport equation for the local residence time of a fluid. Chem. Eng. Sci. 2004, 59, 513-523.

[S5] Lester, E.; Aksomaityte, G.; Li, J.; Gomez, S.; Gonzalez-Gonzalez, J.; Poliakoff, M. Controlled continuous hydrothermal synthesis of cobalt oxide $\left(\mathrm{Co}_{3} \mathrm{O}_{4}\right)$ nanoparticles. Prog. Cryst. Growth Ch. 2012, 58, 3-13. 